\title{
ORIGINAL
}

\section{UTILIZACIÓN DE ANFOTERICINA B NO CONVENCIONAL EN EL HOSPITAL CLÍNICO DE SAN CARLOS}

\author{
Concepción Prieto Yerro (1), Emilio Vargas Castrillón (1), Leonor Laredo Velasco (1), Elisa Pérez- \\ Cecilia (2), María Isabel Ambit Avila (3) y Juan José Picazo de la Garza (2) \\ (1) Servicio de Farmacología Clínica. Hospital Clínico de San Carlos. Madrid. \\ (2) Servicio de Microbiología. Hospital Clínico de San Carlos. Madrid. \\ (3) Servicio de Farmacia. Hospital Clínico de San Carlos. Madrid.
}

\section{RESUMEN}

Fundamento: La anfotericina B es el tratamiento de elección de las infecciones fúngicas sistémicas, pero su utilidad clínica está limitada por su toxicidad. Las formulaciones lipídicas parecen igualmente eficaces y más seguras, pero tienen un mayor coste. El incremento del consumo y del gasto de estas formulaciones nos llevo a plantear un estudio para conocer su perfil de utilización (cuantitativo y cualitativo) y evaluar la repercusión económica de su uso inapropiado.

Métodos: Se desarrollaron unas normas de uso de la anfotericina B y se evaluó de forma retrospectiva, la calidad de la prescripción de la anfotericina $B$ no convencional (anfotericina $\mathrm{B}$ noC) y la repercusión económica de su uso incorrecto.

Resultados: En el $54 \%$ de los tratamientos se hizo una mala selección de anfotericina $B$; en el $3.5 \%$ no estaba indicada la utilización de anfotericina $\mathrm{B}$. El exceso de gasto derivado de la prescripción incorrecta fue de 42 millones de pesetas, un $35 \%$ del gasto total en medicamentos; el gasto por prescripción innecesaria fue de 1.720.327 pesetas.

Conclusiones: La evaluación retrospectiva ha mostrado que existe un elevado porcentaje de tratamientos que no se adecuan a lo recomendado en las normas de prescripción. La puesta en marcha de intervenciones informativas permitiría realizar una selección más eficiente de la anfotericina B noC, mejorando la calidad de la prescripción, lo que podría suponer un importante ahorro economico.

Palabras clave: Anfotericina $B$. Infecciones fúngicas. Estudio de utilización de medicamentos.

\section{Correspondencia:}

Concepción Prieto Yerro

Servicio de Farmacología Clínica

Hospital Clínico de San Carlos

C/ Profesor Martín Lagos sin

28040 Madrid

Correo electrónico: conchapy@ hotmail.com

\section{ABSTRACT \\ Utilisation of non-conventional Amphotericin B in the San Carlos Clinical Hospital}

Background: Amphotericin B is the treatment of choice for systemic fungal infections, however, its clinical usefulness is limited by its toxicity. The lipid formulations appear to be equally effective and safer, but are more costly. The increase in the consumption of, and expenditure on these formulas led us to undertake a study in order to identify their profile of use (quantitative and qualitative) and to assess the financial repercussions when used inappropriately.

Methods: A set of rules were developed for the use of amphotericin B, and the quality of the prescription of nonconventional amphotericin B (amphotericin B notC) was evaluated retrospectively together with the financial repercussions of its inappropriate use.

Results: In $54 \%$ of the treatments studied, a poor selection of amphotericin B was made; in 3.5\%, the use of amphotericin $B$ was not indicated. The excess expenditure derived from the inappropriate use amounted to 42 million pesetas, $35 \%$ of the total expenditure on medicines; the expenditure due to unnecessary prescription was $1,720,327$ pesetas.

Conclusions: The retrospective evaluation has shown that there is a high percentage of treatments that do not conform with the recommendations contained in the prescription rulcs. The holding of information sessions would assist in achieving a more efficient selection of the amphotericin $\mathrm{B}$ notC; this would improve prescription quality, which might also deliver significant financial savings.

Key Words: Amphotericin B. Fungal infections. Study of the use of medicines. 


\section{INTRODUCCIÓN}

Las infecciones fúngicas sistémicas graves son causa de importante morbilidad y mortalidad entre los pacientes inmunodeprimidos (tratados con quimioterapia intensiva, inmunosupresores, enfermos de sida...) y entre los atendidos en unidades de cuidados intensivos. La trascendencia clínica de estas infecciones es aún mayor en los pacientes que se someten a trasplante de médula ósea u otros tipos de trasplantes: en un estudio realizado en 1995 por la European Bone Marrow Transplantation European Organization for Research and Treatment of Cancer ${ }^{1}$ sobre las infecciones fúngicas invasoras en trasplantados de médula ósea en 19 centros europeos, la incidencia de estas infecciones fue del $11.2 \%$, y la mortalidad por candidiasis fue del $58 \%$, por aspergillosis del $76 \%$ y por otras infecciones fúngicas del 54\%.

La anfotericina $B(A B)$ se considera desde los años 50 el tratamiento de elección para la mayoría de estas infecciones, por tener un amplio espectro antifúngico y desarrollar mínimas resistencias; sin embargo, su utilidad clínica se ve limitada tanto por su toxicidad aguda (efectos relacionados con la infusión como fiebre, escalofríos, náuseas, vómitos) como renal, sobre todo en pacientes con función renal alterada y que reciben otros tratamientos nefrotóxicos (ciclosporina, aminoglucósidos, antiinflamatorios no esteroideos, foscarnet... $)^{2-4}$.

Para reducir la toxicidad de la $A B$ se han desarrollado recientemente formulaciones no convencionales ( $\mathrm{AB}$ noC) que asocian la $\mathrm{AB}$ a lípidos ${ }^{5-7}$. En España disponemos de dos de ellas: la $\mathrm{AB}$ unida a verdaderos liposomas $(\mathrm{ABM})$ y la $\mathrm{AB}$ en complejo lipídico (ABL). Los estudios publicados hasta el momento comparan eficacia y seguridad de estos nuevos preparados con la $\mathrm{AB}$ convencional $(\mathrm{ABC})$; $\mathrm{cn}$ ellos las $\mathrm{AB}$ noC parecen tener una eficacia clínica similar a la $A B C$ pero una toxicidad inferior, tanto aguda como renal ${ }^{8-10}$. En 1998 se publicó un estudio retrospectivo observacional en el que se analizó la eficacia y seguridad de ambos preparados de $\mathrm{AB}$ no $\mathrm{C}$ en pacientes hematológicos ${ }^{11}$; no se encontraron diferencias significativas ni en eficacia ni en seguridad, excepto por una mayor proporción de reacciones agudas (tiritonas) en los pacientes que tomaron $A B L$ frente a $A B M$; puesto que se trataba de un estudio observacional y no de un ensayo clínico aleatorizado, los resultados hallados precisaban de confirmación en un estudio experimental bien diseñado. Los dos ensayos clínicos disponibles que comparan las dos $\mathrm{AB}$ no $\mathrm{C}$ en pacientes neutropénicos, no encuentran diferencias estadísticamente significativas en eficacia ${ }^{12-}$ ${ }^{13}$; en lo que se refiere a la seguridad, en uno de ellos tampoco se encontraron diferencias significativas salvo que $\mathrm{ABM}$ se asoció a más alteraciones hepáticas $^{12}$, y el otro mostró mejor perfil de seguridad a favor de $A B M^{13}$. No obstante uno de los trabajos se realizó con una muestra muy pequeña de pacientes y ambos sólo se encuentran disponibles como resumen de congreso. Puesto que no se dispone de ensayos clínicos aleatorizados y ciegos con una muestra grande de pacientes, bien diseñados y publicados que comparen la eficacia y seguridad de las diferentes formulaciones asociadas a lípidos no es posible, de momento, asegurar si un preparado ofrece ventajas frente a otro.

Por otro lado, no todos los pacientes con infección fúngica grave se van a beneficiar de igual manera de la menor toxicidad de las formulaciones asociadas a lípidos, por lo que la elección del tratamiento debería basarse en la valoración individualizada de cada paciente, en especial su estado inmunológico y su función renal ${ }^{14}$.

Los preparados asociados a lípidos tienen además un coste muy elevado, existiendo grandes diferencias entre las $\mathrm{AB}$ disponibles, de modo que el coste/tratamiento/día de la $\mathrm{ABM}$ es aproximadamente 15 veces mayor que el de la $A B C$ y 1,5 veces mayor que el de la $A B L$ (a las dosis habitualmente recomendadas de 5 $\mathrm{mg} / \mathrm{kg} /$ día de ABL y $3 \mathrm{mg} / \mathrm{kg} /$ día de $\mathrm{ABM}$ ). En nuestro hospital, aunque en la práctica el uso de estos preparados está limitado a algunos servicios (Hematología y Oncología fundamentalmente) su utilización supuso en 1999 un coste de 134.057.425 millones de ptas, un 5\% del gasto total en Farmacia. Es necesario, por tanto, que los médicos que atienden a estos pacientes dispongan de herramientas que les per- 
mitan optimizar sus decisiones terapéuticas, no sólo por criterios de eficacia y seguridad sino también de coste-efectividad. Dado el elevado coste sanitario derivado de la atención a los pacientes con infecciones fúngicas sistémicas, así como la gran morbilidad y mortalidad asociada, se plantea el siguiente estudio, con los objetivos de conocer la utilización de la $\mathrm{AB}$ noC ( $\mathrm{ABL}$ y $\mathrm{ABM}$ ) desde un punto de vista cuantitativo y cualitativo y evaluar la repercusión económica de su uso inapropiado en nuestro hospital.

\section{SUJETOS Y MÉTODO}

El estudio se ha realizado en el Hospital Clínico Universitario de San Carlos, entre julio de 1998 y septiembre de 1999. Es un centro con actividad docente que funciona como hospital terciario de referencia; cuenta con 1.100 camas y Servicios de Hematología y Oncología en los que se realizan trasplantes autólogos de médula ósea.

\section{1) Elaboración de las normas de uso de la $\mathrm{AB}$}

En el momento de iniciar el estudio no existían normas explícitas para el uso de antifúngicos en el hospital, por lo que se planteó el trabajo en dos etapas: en la primera y con el fin de disponer de un patrón de referencia se desarrollaron unas «Normas de uso de la $\mathrm{AB}$ en las infecciones fúngicas sistémicas»; en la segunda fase se evaluó, de forma retrospectiva, la concordancia entre la prescripción de la $\mathrm{AB}$ noC y las recomendaciones acordadas, así como la repercusión económica de su utilización. Estas normas, además, podrían servir posteriormente como instrumento para una intervención informativa que permitiera mejorar la concordancia entre la práctica y las recomendaciones recogidas en ellas. Para ello se reunió a un grupo de profesionales del hospital con amplios conocimientos del tema y experiencia en el manejo de los pacientes afectados, pertenecientes a los Servicios de Hematología, Oncología, Medicina Interna, Unidad de Cuidados Intensivos y Farmacia; el grupo de trabajo estuvo coordinado por los Servicios de Farmacología Clínica y Microbiología.
Se realizaron varias reuniones en las que se discutieron las recomendaciones que debían contener las normas de uso. Para ello, se realizó una revisión «no sistemática» de la literatura y la elaboración de las normas se basó en el consenso entre los miembros del grupo de trabajo.

En conjunto las normas sobre la utilización de la $A B$ pueden verse en la tabla 1. La $A B C$ se recomienda en los pacientes no neutropénicos (principalmente pacientes críticos sometidos a ventilación mecánica) con candidiasis sistémica o aspergillosis invasora, infección por $M u c o r$ spp. o Fusarium spp., y con función renal nor$\mathrm{mal}(\mathrm{Cr}<2.5 \mathrm{mg} / \mathrm{dl})$. Si la función renal está alterada o se deteriora de forma progresiva con la utilización de $\mathrm{ABC}$, se recomicnda utilizar ABL. Se considera candidiasis sistémica cuando existe aislamiento microbiológico en líquidos estériles y la colonización por Cándida spp. en al menos tres localizaciones diferentes no estériles en pacientes en situación crítica con sintomatología infecciosa.

En los pacientes neutropénicos tras quimioterapia intensiva o trasplante de médula ósea, en la infección sistémica por Cándida spp. o infección por Aspergillus spp., Mucor spp. o Fusarium spp., así como en el tratamiento empírico del cuadro febril que persiste tras 5 días de tratamiento antibiótico de amplio espectro o 3 días en pacientes en situación crítica, se recomienda utilizar una formulación de $\mathrm{AB}$ noC, ya que se trata de pacientes que toleran mal la toxicidad aguda de $A B C$ y que requieren múltiples tratamientos nefrotóxicos concomitantes. Se recomienda la utilización de $A B L$ por tratarse del preparado de $A B$ noC con mejor relación coste/efectividad.

La utilización de $\mathrm{ABM}$ quedaría restringida a aquellos casos en que el paciente no tolere los efectos indeseables de la $\mathrm{ABL}$ o presente deterioro de la función renal con $\mathrm{Cr}>2.5$ $\mathrm{mg} / \mathrm{dl}$ tras tratamiento con ABL.

\section{2) Evaluación de la calidad de la prescripción de la $\mathrm{AB}$ noC}

Con el fin de conocer la calidad de la prescripción de la $\mathrm{AB}$ noC antes de la difusión de las Normas de uso, se evaluaron los tratamien- 
Tabla 1

Indicaciones recogidas en las Normas de uso de anfotericina B en el Hospital Clínico de San Carlos

\begin{tabular}{|c|c|}
\hline & INDICACIONES CONSENSUADAS \\
\hline $\begin{array}{l}\text { ANFOTERICINA B } \\
\text { CONVENCIONAL (ABC) }\end{array}$ & $\begin{array}{l}\text { - Infección sistémica por Candida spp., otras levaduras o Aspergillus spp. } \\
\text { en pacientes no neutropénicos con función renal normal ( } \mathrm{Cr}<2,5 \mathrm{mg} / \mathrm{dl}) \text {. } \\
\text { - Tratamiento empírico del síndrome febril en pacientes en situación } \\
\text { en situación crítica no neutropénicos que no responden a } 5 \text { días de } \\
\text { tratamiento antibiótico de amplio espectro y con función renal normal } \\
\text { (Cr }<2,5 \mathrm{mg} / \mathrm{dl}) \text {. }\end{array}$ \\
\hline $\begin{array}{l}\text { ANFOTERICINA B } \\
\text { ASOCIADA A LIPIDOS (ABL) }\end{array}$ & $\begin{array}{l}\text { - Las indicaciones del apartado anterior cuando la función renal está } \\
\text { alterada }(\mathrm{C}>2,5 \mathrm{mg} / \mathrm{dl}) \text {. } \\
\text { - Intolerancia a la } \mathrm{ABC} \text { en las indicaciones del apartado anterior. } \\
\text { - Infección sistémica por Candida spp., otras levaduras o Aspergillus spp. } \\
\text { en pacientes neutropénicos, independientemente de la función renal. } \\
\text { - Tratamiento empírico del síndrome febril en pacientes neutropénicos } \\
\text { que no responden a 3-5 días de tratamiento antibiótico de amplio espectro. }\end{array}$ \\
\hline $\begin{array}{l}\text { ANFOTERICINA B EN } \\
\text { COMPLEJO LIPOSOMAL (ABM) }\end{array}$ & $\begin{array}{l}\text { - Las indicaciones del apartado anterior que presenten deterioro de la } \\
\text { función renal o intolerancia tras la administración de } A B L \text {. }\end{array}$ \\
\hline
\end{tabular}

tos prescritos con esta $\mathrm{AB}$ en el periodo de estudio, utilizándose las Normas anteriormente citadas como patrón de referencia.

Para delimitar el marco muestral se definió como unidad de estudio cada tratamiento con $\mathrm{AB}$ noC prescrito en el hospital durante el periodo comprendido entre el 1 de julio de 1998 y el 15 de septiembre de 1999, independientemente del diagnóstico. La información básica sobre cada tratamiento (nombre del paciente, tipo de $\mathrm{AB}$ noC prescrita, dosis y duración del tratamiento) fue facilitada por el Servicio de Farmacia del Hospital, así como el coste/mg (en pesetas).

Para conocer la indicación de cada tratamiento y los datos clínicos necesarios para evaluar la calidad de la misma, se utilizaron las historias clínicas como fuente de datos. Se recogió en una hoja especialmente diseñada para ello, la siguiente información: datos del paciente, servicio que prescribe, tipo de $\mathrm{AB}$ noC prescrita, adecuación de la prescripción según las indicaciones consensuadas, dosis prescrita (en $\mathrm{mg}$ /día) y duración del tratamiento (en días).
Los datos se introdujeron en una base de datos de programa ACCESS 97 y se analizaron con el programa SPSS 8.0 de Windows. Se realizó un análisis descriptivo de las variables de interés. Además se cuantificó el gasto derivado de la prescripción de la $\mathrm{AB}$ noC, así como la repercusión económica del exceso de gasto producido como consecuencia del uso incorrecto de la $\mathrm{AB}$. Se consideró «incorrecta» cualquier indicación no reconocida como tal en la literatura biomédica o no incluida en las Normas de Uso consensuadas y cualquier tratamiento en el que se prescribió una $\mathrm{AB}$ estando recomendada otra (por ej. la $\mathrm{ABM}$ estando recomendada la $\mathrm{ABL}$ o la $\mathrm{ABC}$ ).

\section{RESULTADOS}

\section{1) Evaluación cualitativa y cuantitativa de la prescripción de $\mathrm{AB}$ noC}

El análisis descriptivo muestra que se prescribieron un total de 245 tratamientos con $\mathrm{AB}$ noC; en el $53 \%$ se utilizó ABL y en el $47 \%$ 
ABM. Del total, 184 tratamientos (el 75\%) fueron prescritos por los Servicios de Ilematología y Oncología (tabla 2).

La indicación para la que se prescribió la $\mathrm{AB}$ noC con más frecuencia fue el tratamiento empírico del síndrome febril en pacientes neutropénicos $(36 \%)$, seguida de la profilaxis de aspergillosis en este mismo grupo de pacientes $(29 \%)$ (tratamiento para el que se prescribió la $\mathrm{AB}$ noC debido a la existencia de las obras de acondicionamiento del hospital).

Se consideraron incorrectas 170 prescripciones, de éstas 35 (el 21\%) se indicaron para el tratamiento empírico del síndrome febril mantenido en pacientes neutropénicos tras 3-5 días de administración con antibióticos de amplio espectro y 24 (el 14\%) para la sospecha de infección por Mucor spp., Fusarium spp. o aspergillosis invasora. En cuatro casos fue imposible determinar la indicación a partir de los datos disponibles en la historia clínica (tabla 3).

De estos tratamientos considerados incorrectos, $6(3.5 \%)$ resultaron ser innecesarios por no estar indicada la utilización de $A B$; en 92

Tabla 2

Tratamientos prescritos con anfotericina B no convencional y su porcentaje sobre el total, por servicios

\begin{tabular}{|lccc|}
\hline Servicio & $A B L$ & $A B M$ & Total \\
\hline Hematología & 83 & 57 & 140 \\
Oncología & 30 & 14 & 44 \\
Pediatría & 0 & 9 & 9 \\
UCI & 14 & 9 & 23 \\
M. Interna & 2 & 20 & 22 \\
Nefrología & 0 & 5 & 5 \\
Cirugía & 0 & 2 & 2 \\
\hline Total (\%) & $129(53)$ & $116(47)$ & 245 \\
\hline
\end{tabular}

ABL: anfotericina $\mathrm{B}$ lipídica

ABM: anfotericina B liposomal

UCI: Unidad de cuidados Intensivos

M. Interna: Medicina Interna
(54\%) estaba indicado prescribir $\mathrm{AB}$ noC pero se realizó una mala selección de la misma, fundamentalmente de ABM. Los 72 tratamientos restantes se refieren a los pautados para profilaxis de infección por Aspergillus spp. (tabla 4).

\section{2) Evaluación del coste del uso de $\mathrm{AB}$ noC y del exceso de gasto derivado de la prescripción inadecuada de la misma}

En la tabla 5 se muestra el exceso de gasto derivado de la prescripción incorrecta de $\mathrm{AB}$ noC, casi 42 millones y medio de pesetas que representan un $35 \%$ del gasto total en esta medicación (120 millones) en el periodo de estudio. El 88\% del exceso de gasto (37 millones) se debe a la selección inadecuada de $\mathrm{ABM}$ en pacientes que podrían haber sido manejados con $\mathrm{ABL}$ o $\mathrm{ABC}$, y el $12 \%$ (5 millones) a la selección de $\mathrm{ABL}$ en pacientes que no requerían ninguna anfotericina (aquí incluimos el debatido uso profiláctico en pacientes neutropénicos) o que podrían haber sido manejados con $\mathrm{ABC}$. El exceso de gasto producido por la prescripción innecesaria de $\mathrm{AB}$ noC fue de 1.720 .327 pesetas y el derivado de la prescripción de $A B$ noC para la profilaxis de la aspergillosis fue de alrededor de 3 millones de pesetas ( $7 \%$ sobre el total).

\section{DISCUSIÓN}

El uso de la $\mathrm{AB}$ noC (ABL yABM) se ha incrementado notablemente en los últimos años en nuestro hospital. El desarrollo de las formulaciones de $\mathrm{AB}$ asociadas a lípidos $(\mathrm{AB}$ en complejo lipídico y $\mathrm{AB}$ en complejo liposomal) ha determinado además un cambio en el patrón de utilización de $\mathrm{AB}$ y una considerable variabilidad interpráctica a la hora de seleccionar la $\mathrm{AB}$. En nuestro estudio se demuestra que, globalmente y desde una perspectiva clínica, el uso de $\mathrm{AB}$ noC fue adecuado pero poco eficiente.

En el desarrollo de nuestro trabajo hemos encontrado varias limitaciones; en primer lugar, la falta de ensayos clínicos comparativos sobre eficacia y seguridad de las dos formulaciones de $\mathrm{AB}$ asociadas a lípidos no nos 
Tabla 3

Adecuación de la prescripción de anfotericina B no convencional, según la indicación

\begin{tabular}{|c|c|c|c|c|}
\hline Indicación & Incorrecta & Correcta & No concluyente & Total \% \\
\hline $\begin{array}{l}\text { Candidiasis con aislamiento microbiológico en líquidos } \\
\text { estériles (sangre, LCR, líquido peritoneal) en neutropénicos }\end{array}$ & 2 & 0 & 0 & $2(0,8)$ \\
\hline $\begin{array}{l}\text { Candidiasis con aislamiento microbiológico en líquidos } \\
\text { estériles (sangre, LCR, líquido peritoneal) en no } \\
\text { neutropénicos }\end{array}$ & 6 & 2 & 0 & $8(3,3)$ \\
\hline $\begin{array}{l}\text { Colonización por Candida spp. en menos de tres } \\
\text { localizaciones no esteriles }\end{array}$ & 6 & 0 & 0 & $6(2,5)$ \\
\hline $\begin{array}{l}\text { Colonización por Candida spp. en al menos tres } \\
\text { localizaciones no cstériles en pacientes en situación } \\
\text { crítica con sintomatología infecciosa }\end{array}$ & 1 & 1 & 0 & $2(0,8)$ \\
\hline $\begin{array}{l}\text { Aspergillosis diagnosticada por presencia de } \\
\text { Aspergillus spp. en catéter telescopado más Rx tórax } \\
\text { compatible (en neutropénicos) }\end{array}$ & 5 & 0 & 0 & $5(2)$ \\
\hline $\begin{array}{l}\text { Tratamiento empírico (Aspergillus spp. o levaduras) en } \\
\text { cuadro febril tras cinco días de antibiótico amplio espectro } \\
\text { en pacientes en situación crítica (no neutropénicos) }\end{array}$ & 16 & 6 & 0 & $22(9)$ \\
\hline $\begin{array}{l}\text { Tratamiento empírico (Aspergillus spp. o levaduras) en } \\
\text { cuadro febril tras cinco días de antibióticos de amplio } \\
\text { espectro en pacientes en situación crítica (neutropénicos) }\end{array}$ & 35 & 53 & 0 & $88(35,9)$ \\
\hline Aislamiento de Candida no albicans, C. krusei o C. glabrata & 3 & 2 & 0 & $5(2)$ \\
\hline $\begin{array}{l}\text { Sospecha de infección por Mucor spp., Fusarium spp. o } \\
\text { aspergillosis invasiva }\end{array}$ & 24 & 7 & 0 & $31(12,7)$ \\
\hline $\begin{array}{l}\text { Profilaxis de infección por Aspergillus spp. en pacientes } \\
\text { neutropénicos graves }\end{array}$ & 72 & 0 & 0 & $72(29,4)$ \\
\hline Indicación desconocida & 0 & 0 & 4 & $4(1,6)$ \\
\hline TOTAL (\% sobre total) & $170(69)$ & $71(29)$ & $4(2)$ & 245 \\
\hline
\end{tabular}

LCR: líquido cefalorraquídeo.

$\mathrm{RX}$ : radiografía.

permite conocer si un preparado es mejor que otro. Los estudios que comparan una de las dos formulaciones con $\mathrm{ABC}$ han demostrado que ambas tienen una eficacia similar a la $\mathrm{ABC}$ con una menor incidencia de efectos secundarios $^{8-10}$. Los dos ensayos clínicos disponibles que comparan las dos $\mathrm{AB}$ noC no encucntran diferencias estadísticamente significativas en eficacia ${ }^{12,13}$ y son contradictorios en cuanto al perfil de seguridad, por lo que sería necesario contar con otros estudios realizados con un tamaño de muestra grande y bien diseñados. Asumiendo la escasa información disponible y teniendo en cuenta que el coste/tratamiento/día de ABM es 1.5 más elevado que el de $\mathrm{ABL}$, utilizándolos a las dosis habitualmente recomendadas en la ficha técnica de cada preparado $(5 \mathrm{mg} / \mathrm{kg} / \mathrm{día}$ de $\Lambda \mathrm{BL}$ y $3 \mathrm{mg} / \mathrm{kg} /$ día de $\mathrm{ABM}$ ), hemos considerado que $\mathrm{ABL}$ sería el preparado con una mejor relación coste/efectividad y por lo tanto la $\mathrm{AB}$ noC de elección. 
Tabla 4

Tratamientos incorrectos prescritos con anfotericina B no convencional

\begin{tabular}{|lccc|}
\hline & $A B L$ & $A B M$ & Total \\
\hline $\begin{array}{l}\mathrm{N} .{ }^{0} \text { Total de tratamientos } \\
\text { incorrectos } \\
\begin{array}{l}\text { - Profilaxis de infección por } \\
\text { Aspergillus spp. }\end{array}\end{array}$ & 52 & 98 & 170 \\
$\begin{array}{l}\text { Seleccióno inadecuada de } \\
\text { la AB noC }\end{array}$ & 7 & 85 & 92 \\
- Innecesarios & 6 & - & 6 \\
\hline
\end{tabular}

ABL: anfotericina $B$ lipídica. ABM: anfotericina B liposomal. $A B$ noC: anfotericina $B$ no convencional.

Por otro lado, la principal limitación de la metodología utilizada para definir las indicaciones de la $\mathrm{AB}$ noC ha sido que la revisión de la literatura no fue sistemática y, por lo tanto, pudo estar sujeta a sesgos en la interpretación de los resultados. No obstante, el procedimiento se basó en el consenso y en la participación de todos los servicios implicados en la prescripción de $\mathrm{AB}$, lo que habitualmente debería garantizar una amplia aceptación de las normas por parte de los prescriptores.
La obtención de los datos a partir de la historia clínica ha sido otra limitación de nuestro trabajo. Aunque los estudios sobre calidad de la asistencia realizados en el medio hospitalario y extrahospitalario indican que cada vez se registran más datos en la historia clínica ${ }^{15}$, el elevado porcentaje de tratamientos inadecuados encontrados en nuestro trabajo, hace pensar en la posibilidad de que haya existido un sesgo de mala clasificación de los tratamientos. Dado que éstos se han clasificado como «correctos» o «incorrectos» de acuerdo a los datos registrados en la historia, es posible que la falta de algunos datos clínicos haya sobreestimado el porcentaje de tratamientos inadecuados. Sin embargo, creemos que este sesgo no invalidaría nuestros hallazgos dado que se necesitaría un número de tratamientos mal clasificados muy elevado para modificar el sentido de los resultados. No obstante, el registro cuidadoso de los efectos secundarios relacionados con la $\mathrm{AB}$ hubiera sido fundamental para justificar la selección de una $A B$ noC en muchos casos. Los médicos deberían ser conscientes de la importancia de que este aspecto depende sólo de ellos y por tanto es fácilmente mejorable.

También debe tenerse en cuenta que entre los tratamientos considerados incorrectos se encuentran aquellos casos en los que la $\mathrm{AB}$ noC se prescribió a dosis bajas en pacientes

Tabla 5

Exceso de gasto derivado de la prescripción incorrecta de anfotericina $\mathbf{B}$ no convencional

\begin{tabular}{|lccc|}
\hline & ABL & $A B M$ & Total \\
\hline $\begin{array}{l}\text { 1. Exceso de gasto derivado de la prescripción en profilaxis } \\
\text { de Aspergillus, spp. en ptas. }\end{array}$ & 1.959 .990 & 1.424 .113 & 3.384 .103 \\
$\begin{array}{l}\text { 2. Exceso de gasto derivado de la selección incorrecta de } \\
\text { AB noC, en ptas. }\end{array}$ & 1.440 .417 & 35.923 .911 & 37.364 .328 \\
$\begin{array}{l}\text { 3. Exceso de gasto derivado de los tratamientos innecesarios, } \\
\text { en ptas. }\end{array}$ & 1.720 .327 & & 1.720 .327 \\
\hline Exceso total de gasto, en ptas. & 5.120 .734 & 37.348 .024 & 42.468 .758 \\
\hline
\end{tabular}

AB noC: anfotericina $B$ no convencional.

ABL: anfotericina $B$ lipídica.

ABM: anfotericina B liposomal. 
neutropénicos para la cuestionada indicación de profilaxis de la infección por Aspergillus spp. ${ }^{16-18}$. Es importante comentar que durante el primer semestre de 1998 se realizaron obras de reformas en el hospital y que éstas se han señalado en algunos estudios como factor de riesgo de infecciones por Aspergillus spp. en los pacientes neutropénicos ${ }^{19,20}$; por ello se acordó que el servicio de hematología prescribiría $\mathrm{AB}$ noC temporalmente a los pacientes neutropénicos como profilaxis de la infección por este microorganismo; esta situación se mantuvo hasta mayo del 99 , momento a partir del cual dejó de recetarse para esta indicación. Aunque el número de tratamientos prescritos para profilaxis de infección por Aspergillus spp. fue elevado, el coste derivado de los mismos fue realmente pequeño.

El exceso de gasto derivado de la prescripción inadecuada hace referencia al ahorro que se podría haber obtenido si sólo se hubieran realizado los tratamientos correctos. Se incluye en este grupo el exceso de gasto debido a una selección incorrecta de la $\mathrm{AB}$ noC, el que se ha debido a la prescripción coyuntural de $\mathrm{AB}$ noC como profilaxis de la infección por Aspergillus spp. y el que se ha producido como consecuencia de la prescripción innecesaria de $\mathrm{AB}$ noC. Llama la atención que la mayor parte de este exceso de gasto (más de 37 millones) se debe a la selección inadecuada de la ABM; esto sugiere que la puesta en marcha de intervenciones de carácter informativo sobre los prescriptores permitiría realizar una selección más eficiente de la $\mathrm{AB}$ noC y podría suponer un ahorro potencial de más de 30 millones de pesetas el próximo año, si la evolución del gasto permaneciera constante.

No hemos encontrado estudios publicados en la literatura que nos permitan establecer comparaciones con los resultados obtenidos en nuestro trabajo, pero creemos que se encontraría también un incremento de consumo de $\mathrm{AB}$ y una distribución similar entre las dos formulaciones disponibles de $\mathrm{AB}$ noC.

Los resultados de nuestro estudio indican que la calidad de la prescripción de la $\mathrm{AB}$ noC no ha sido óptima. La falta de unas normas de uso explícitas es probablemente una de las causas que explican las diferencias de criterio encontradas; cl desarrollo de las normas de utilización de $\mathrm{AB}$ y su divulgación pueden contribuir a racionalizar la prescripción y a reducir el coste innecesario.

\section{BIBLIOGRAFÍA}

1. EORTC International Antimicrobial Therapy Cooperative Group. Empirical antifungal therapy in granulocytopenic patients. Am J Med 1989; 86: 668-72

2. Criteria for use of amphotericina B lipid complex injection in adults and children. Am J Health Sys Pharm 1996; 53 (15): 2751-2.

3. Tollemar J, Ringden $\mathrm{O}$. Lipid formulations of amphotericin B. Less toxicity but at what economic cost? Drug Safety 1995; 13 (4): 207-18.

4. Sugar AM. Empiric treatment of fungal infections in the neutropenic host. Review of the literature and guidelines for use. Arch Intern Med 1990; 150 (11): 2258-64

5. Hemenz JW, Walsh TJ. Lipid formulations of amphotericin B: recent progress and future directions. Clin Inf Dis 1996; 22 (2): 133-44.

6. Graybill JR. Lipid formulations for amphotericin B: Does the emperor need new clothes?. Ann Intern Med 1996; 124 (10): 921-3.

7. Coukell AJ, Brogden RN. Liposomal Amphotericin B. Therapeutic use in the management of fungal infections and visceral leishmaniasis. Drugs 1998; 55 (4): 585-612.

8. Tierney MG, Grunet AM, McLean WM, Toye BW, Garber GE. Liposomal amphotericin B: a cost-outcome analysis. Can J Hosp Pharm 1994; 47 (4): 171-5

9. Moreau P, Milpied N, Fayette N, Ramée JF, Harousseau JL. Reduced renal toxicity and improved clinical tolerance of amphotericin B mixed with intralipid comparcd with conventional amphotericin $B$ in neutropenic patients. J Antimicrob Chemother 1992, 30 (4): 535-41

10. White MH, Bowden RA, Sandler ES, Graham ML, Noskin GA, Wingard JR et al. Randomized, double-blind clinical trial of amphotericin B colloidal dispersion versus amphotericin B in the empirical treatment of fever and ncutropenia. Clin Infect Dis 1998; 27 (2): 296-302 
11. Clark AD, Mckendrick S, Tansey PJ, Franklin IM, Chopra R. A comparative analysis of lipid-complexed and liposomal amphotericin $\mathrm{B}$ preparations in haematological oncology. $\mathrm{Br}$ J Haematol 1998; 103: 198-204.

12. Fleming R, Kantajian H, Husni R, Anaissie E, Smith T, Cortes J et al. Randomized study of two lipid formulation of Amphotericin B in the treatment of suspected or documented fungal infections in patients with leukemia. San Diego: 9th Annual Focus on Fungal Infections Meeting; 1999.

13. Wingard JR, White MH, Anaissie EJ, Rafalli JT, Goodman JL, Arrieta AC. A randomized double-blind comparative safety trial of Ambisome and Abelcet in febrile neutropenic patients. San Diego: 9th Annual Focus on Fungal Infections Meeting; 1999.

14. Pahissa A. Anfotericina B. Complejo lipídico versus liposómica ¿cuál, por qué, cuando?. Enferm Infecc Microbiol Clin 1997; 15: 1-3.

15. Saturno PJ. Monitorización del uso de medicamentos para mejorar la calidad de la prescripción. Problemas, métodos e indicadores. Aten Primaria 1996; 18 (6): 331-9
16. Lamm HH, Althaus BL. Antifungal prophyla$x$ is in bone marrow transplant. Ann Pharmacother, 1995 Sep, 29: 9, 921-4

17. Gubbins PO, Bowman JL, Penzak SR. Antifungal prophylaxis to prevent invasive mycoses among bone marrow transplantation recipients. Pharmacotherapy 1998; 18 (3): 549-64

18. Rousey SR, Russler S, Gottlieb M, Ash RC Low-dose amphotericin B prophylaxis against invasive Aspergillus infections in allogeneic marrow transplantation. Am J Med 1991; 91 (5): 484-92

19. Weems JJ, Davis BJ, Tablan OC, Kaufman L, Martone WJ. Construction activity: an independent risk factor for invasive aspergillosis and zygomycosis in patients with hematologic malignancy. Infect Control 1987; 8 (2): 71-5

20. Weber SF, Peacock JE, Do KA; Cruz JM; Powell BL; Capizzi RL. Interaction of granulocytopenia and construction activity as risk factors for nosocomial invasive filamentous fungal disease in patients with hematologic disorders. Infect Control Hosp Epidemiol 1990; 11 (5): 235-42. 